\title{
Salmeterol/fluticasone combination instead of indacaterol or vice-versa?
}

To the Editor:

The Indacaterol: Switching Non-exacerbating Patients with Moderate COPD From Salmeterol/Fluticasone to Indacaterol (INSTEAD) study, published in the December edition of the European Respiratory Journal, concluded that that "patients with moderate COPD and no exacerbations in the previous year can be switched from salmeterol/fluticasone (SFC) to indacaterol with no efficacy loss" [1]. We would like to respectfully challenge this conclusion (as suggested also by the acronym "INSTEAD") since, in our opinion, the data presented do not support it. First, the study was designed and powered to address a short-term (12 weeks), physiological question (the primary outcome was non-inferiority of lung function changes (through forced expiratory volume in $1 \mathrm{~s}\left(\mathrm{FEV}_{1}\right)$ ). This study design does not allow any inference about the relative clinical positioning of these two therapeutic alternatives at longer term (daily control or future risk, e.g. exacerbation, progression of disease, or mortality). Secondly, authors included in the study "patients receiving SFC 50/500 $\mathrm{mg}$ for $\geqslant 3$ months, with no chronic obstructive pulmonary disease (COPD) exacerbations for $>1$ year before the study (patients for whom inhaled corticosteroid (ICS) is not recommended)". In our opinion, their interpretation that ICS are not recommended in these patients is both arguable and relevant. Arguable, because authors cannot disregard the possibility that patients did not have exacerbations in the previous year precisely because they were treated with ICS. Relevant because if these patients did not really need ICS, then it should not surprise that ICS withdrawal was "non-inferior" to bronchodilator treatment. Finally, based on the INSTEAD results, the exact opposite conclusions might be reached. This is, given that: 1) treatment with indacaterol is not inferior to treatment with SFC in terms of lung function (primary outcome), and/or daily control (symptoms, quality of life); and, 2) there is no information on the long-term effects of indacaterol on the rate of exacerbations, whereas this is well established for the SFC combination in a properly designed and longer-term study [24], the latter might be favoured over indacaterol. It goes without saying that both conclusions (this one and that of the INSTEAD study) are speculative, and thus properly designed and powered studies are required before any evidence based recommendation on the use of ICS in COPD can be reliably made [57]. In any case, we congratulate the authors of the INSTEAD study for their interesting work that contribute to another piece of evidence on the debate of when to use ICS in COPD. However, we honestly believe that the clinical over-interpretation of their results may not be in the best interest of patients, since there is a group of patients with COPD who can clearly benefit from ICS in combination with long-acting bronchodilators, including those with repeated exacerbations (despite adequate bronchodilator treatment) and the asthma-COPD overlap syndrome [8].

@ERSpublications

Lung function and relevance on choice of indacaterol or salmeterol/fluticasone combination in moderate COPD patients http://ow.ly/HGOCV

Leonardo M. Fabbri ${ }^{1}$ and Alvar Agusti ${ }^{2,3}$

${ }^{1}$ Respiratory Disease Unit, University of Modena and Reggio Emilia, Modena, Italy. ${ }^{2}$ Hospital Clinic de Barcelona, CIBER en Enfermedades Respiratorias (CIBERES), Barcelona, Spain. ${ }^{3}$ Institut d'Investigacions Biomèdiques August Pi i Sunyer (IDIBAPS), Universitat de Barcelona, Barcelona, Spain.

Correspondence: Leonardo M. Fabbri, University of Modena and Reggio Emilia,Respiratory Disease Unit, Pozzo 71, Modena, Italy. Email: leonardo.fabbri@unimore.it

Received: Dec 102014 | Accepted: Dec 112014

Conflict of interest: Disclosures can be found alongside the online version of this article at erj.ersjournals.com

References

1 Rossi A, van der Molen T, Olmo RD, et al. INSTEAD: a randomised switch trial of indacaterol versus salmeterol/ fluticasone in moderate COPD. Eur Respir J 2014; 44: 1548-1556.

2 Calverley PM, Anderson JA, Celli B, et al. Salmeterol and fluticasone propionate and survival in chronic obstructive pulmonary disease. $N$ Engl J Med 2007; 356: 775-789. 
3 Celli BR, Thomas NE, Anderson JA, et al. Effect of pharmacotherapy on rate of decline of lung function in chronic obstructive pulmonary disease: results from the TORCH study. Am J Respir Crit Care Med 2008; 178: 332-338.

4 Jenkins CR, Jones PW, Calverley PM, et al. Efficacy of salmeterol/ fluticasone propionate by GOLD stage of chronic obstructive pulmonary disease: analysis from the randomised, placebo-controlled TORCH study. Respir Res 2009; 10: 59.

5 National Institute for Health and Clinical Excellence (NICE). Chronic Obstructive Pulmonary Disease. Management of Chronic Obstructive Pulmonary Disease in Adults in Primary and Secondary Care. NICE, 2010. Available from: www.nice.org.uk/guidance/cg101

6 Qaseem A, Wilt TJ, Weinberger SE, et al. Diagnosis and management of stable chronic obstructive pulmonary disease: a clinical practice guideline update from the American College of Physicians, American College of Chest Physicians, American Thoracic Society, and European Respiratory Society. Ann Intern Med 2011; 155: 179-191.

7 Global Initiative on Obstructive Lung Disease. Global Strategy for Diagnosis Management and Prevention of Chronic Obstructive Pulmonary Disease. Global Initiative on Obstructive Lung Disease, 2014. Available from: www. goldcopd.org/guidelines-global-strategy-for-diagnosis-management.html

8 Agusti A, Fabbri LM. Inhaled steroids in COPD: when should they be used? Lancet Respir Med 2014; 2: 869-871.

Eur Respir J 2015; 45: 1187-1188 | DOI: 10.1183/09031936.00226314 | Copyright @eERS 2015

From the authors

We read with interest the comments by L.M. Fabbri and A. Augusti on our article entitled "INSTEAD: a randomised switch trial of indacaterol versus salmeterol/fluticasone in moderate COPD" [1]. However, we believe that those comments go well beyond the conclusion of the study, which is well supported by the data.

The Indacaterol: Switching Non-exacerbating Patients with Moderate COPD From Salmeterol/Fluticasone to Indacaterol (INSTEAD) trial examined a population of chronic obstructive pulmonary disease (COPD) patients with moderate airflow limitation [2], without a history of exacerbations in the previous year, and on treatment with inhaled corticosteroids (ICS)/long-acting $\beta$-agonist (LABA) combination therapy for $\geqslant 3$ months. After randomisation, 250 patients continued and completed 6-month treatments with salmeterol/ fluticasone fixed-dose combination (SFC), while 246 patients completed 6-month treatments with indacaterol monotherapy, after switch from SFC without a washout period. The study data showed no difference in lung function (forced expiratory volume in $1 \mathrm{~s}$ (FEV1) in the whole population and inspiratory capacity in a subgroup), symptoms, quality of life or exacerbations between the two arms and the two therapy regimens. The conclusion of the study, which is difficult to challenge, was that the population of COPD patients in the INSTEAD study does not need ICS, and that ICS can be safely withdrawn, if that treatment was initiated. In fact, according to COPD management guidance, these patients should not receive ICS in addition to their maintenance treatment with long-acting bronchodilators [3]. The results of our study should be evaluated for the recruited population and not for patients with either more severe COPD or frequent exacerbations, or both. It is important to emphasise that INSTEAD excluded COPD patients with severe airflow limitation (FEV1 $<50 \%$ predicted) and patients with exacerbations in the previous year, i.e. grades III and IV [2]. Clearly, any history of either asthma or like-asthma symptoms was in the exclusion criteria [1]. The INSTEAD randomised trial confirms the results of a recent real-life study on $>800$ moderate COPD patients [4], and supports the current management guidance on moderate COPD without history of exacerbations [3]. Were the patients in INSTEAD exacerbation-free in the previous year because they were treated with ICS? It seems very unlikely, because they did not exacerbate when ICS was withdrawn. After careful reading we do not see any significant discrepancy between the conclusion of INSTEAD and the recommendation of a recent editorial [5]. Clearly, the appropriate use of ICS in COPD is an important issue [5]. In fact, the possible benefit of ICS on airway inflammation [6] and exacerbation rate was found to be associated with side-effects [7, 8].

Any speculation on longer-term effects of indacaterol, and on the impact of ICS withdrawal in severe COPD patients with history of exacerbations, goes well beyond the purpose, the design, and the data of the INSTEAD study, but is explored in other trials $[9,10]$. Likewise, we do not make any over interpretation either of the potential benefits of ICS in severe "frequent exacerbators" COPD patients [11] or on the use of the ICS/LABA combination in patients with the so called "asthma-COPD overlap syndrome" [5], although it might be a very interesting debate $[12,13]$.

We believe that INSTEAD data provides a significant contribution to the debate on the appropriate use of ICS in COPD patients $[3,14]$. 